\title{
TECHNIQUE AND INTERPRETATION OF SELECTIVE CORONARY ARTERIOGRAPHY IN MAN
}

\author{
BY \\ GEORGE HALE* AND KEITH JEFFERSON \\ From St. George's Hospital, London S.W.1
}

Received January 11, 1963

The radiographic study of coronary arteries in man has been attempted only in recent years. The earliest workers in this sphere employed thoracic aortography, the opaque medium being introduced by needle puncture (Radner, 1945) or retrograde catheterization of the ascending aorta. Lehman, Boyer, and Winter (1959) have reviewed the early results and the subsequent development of aortography, and it was apparent that aortography alone was unsatisfactory because coronary artery opacification was not regularly achieved. Thus in a series studied by catheter thoracic aortography (Lehman et al., 1959) no visualization of either coronary artery was obtained in 10 of the 44 patients. Many modifications have been devised to improve coronary artery filling but the hazards of the investigation are thereby increased. Most methods described have involved temporary reduction of the cardiac output during the injection of contrast medium. Thus Dotter and Frische (1958) employed balloon occlusion of the aorta; and Arnulf and Buffard (1959), Gensini, Di Giorgi, and Black (1961), and Bilgutay and Lillehei (1962) induced bradycardia or cardiac arrest with acetylcholine. Nordenström, Ovenfors, and Törnell (1962) used general anæsthesia with aortography and by increasing intrabronchial pressure to about $40 \mathrm{~cm}$. of water they achieved a drop in aortic systolic blood pressure to about $70 \mathrm{~mm}$. $\mathrm{Hg}$. This technique appears to be safe and the published radiographs show excellent coronary opacification. A loop-end polyethylene catheter has been designed by Williams et al. (1960), the aim being to fill the aortic sinuses so that a high proportion of the medium enters the coronary arteries. In our animal experiments we found that cardiac arrest induced by acetylcholine (Sloman and Jefferson, 1960) and timed diastolic injections of contrast medium (Michell and Jefferson, 1962) gave encouraging results, but we considered that cardiac arrest was potentially too dangerous to use in man. Although Thal et al. (1958) have applied timed diastolic injections successfully in man, our experience convinced us that it was unreliable in filling diseased coronary arteries. Failure to fill a severely diseased right coronary artery is illustrated by Case 4 (Fig. 1 and 2). This patient, a man aged 44, had had severe angina of effort for five years. Aortography with $75 \mathrm{ml}$. opaque medium showed occlusion of the anterior descending artery but failed to outline the right coronary artery (Fig. 1). Selective coronary arteriography was subsequently performed, and the right coronary artery was successfully cannulated and clearly outlined by an injection of only $2 \mathrm{ml}$. (Fig. 2).

Thus, in 1960, faced with the problem of how to improve aortography without increasing the hazards of the procedure, we decided to investigate selective coronary arteriography, introduced by Sones in 1960 and practised by him with success and safety. Apart from Sones, who has had an extensive experience with selective coronary arteriography (Sones and Shirley, 1962), Lang and Sabiston (1961) and Tapia, Bolton, and Mazel (1961) have also reported favourably.

* In receipt of Medical Research Council Grant. Present address: St. Vincent's Hospital, Melbourne, N.6., Australia. 




Fig. 1.-Case 4. Aortography using $75 \mathrm{ml} .76$ per cent urografin divided into three doses and injected during three successive diastolic periods. Aortogram with a patient in a $10^{\circ}$ right anterior oblique position. Severe narrowing of the left anterior descending artery is demonstrated but there is no opacification of the right coronary artery.

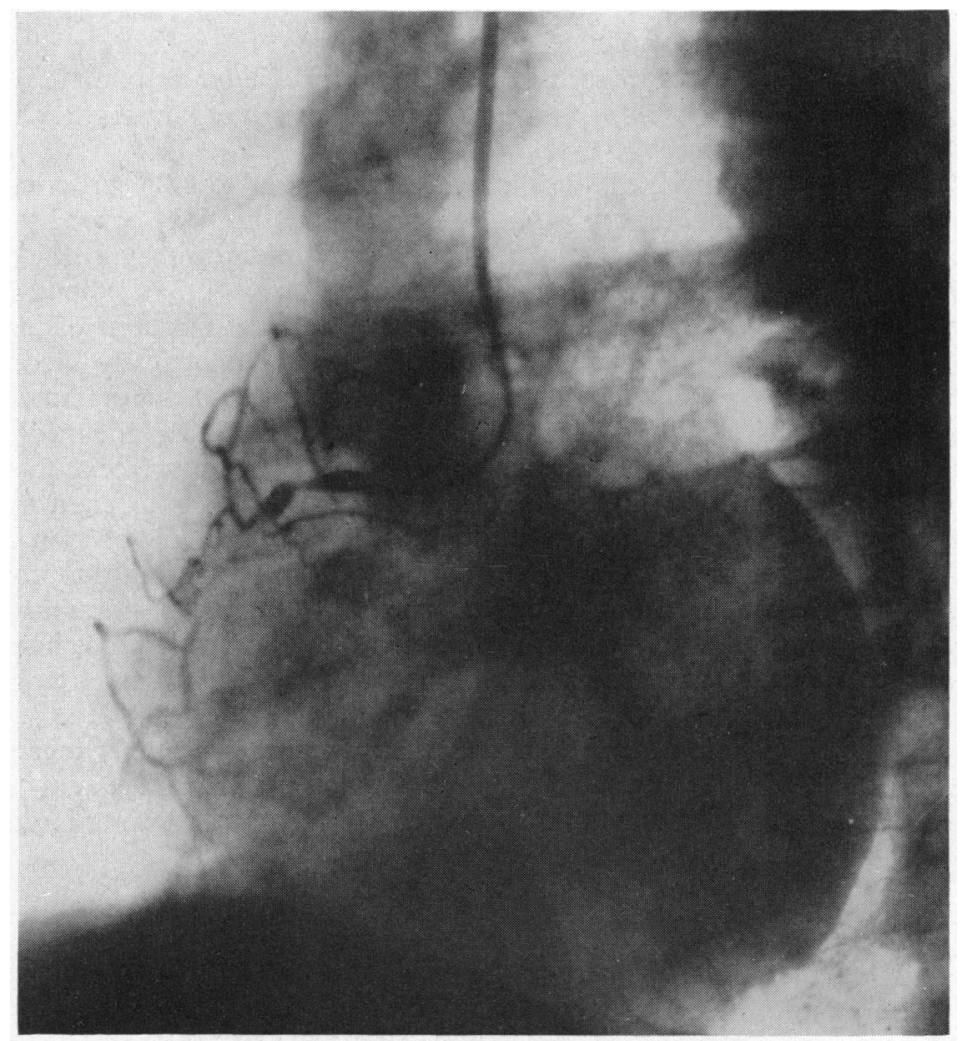

Fig. 2.-Case 4. Selective coronary injection of $2 \mathrm{ml}$. contrast medium into the right coronary artery with single plate $\mathrm{x}$-ray. Patient in left anterior oblique position. A complete block $1 \mathrm{~cm}$. from its origin is demonstrated, with collateral channels filling the distal part of the artery. 


\section{SubJECTS AND METHODS}

A total of 25 patients have been studied by selective coronary arteriography during the past two years. The reasons for investigating these patients have included incapacitating angina demanding surgical relief if there were localized disease amenable to endarterectomy, occasionally in patients with angina and valve disease amenable to surgery if advanced coronary disease was absent, and occasionally in patients with incapacitating chest pain of uncertain origin. A report on the findings, including a discussion of the value of coronary arteriography in clinical practice, is being prepared. Before coronary arteriography, all patients were assessed clinically. Electrocardiography was performed at rest and with exercise using a bicycle ergometer. In those patients with valvular heart disease, the appropriate left and right heart catheter investigations were performed at the time of selective coronary arteriography.

The Technique of Selective Coronary Arteriography. A premedication such as a combination of papaveratum 10 to $20 \mathrm{mg}$. by subcutaneous injection, quinalbarbitone sodium 100 to $200 \mathrm{mg}$., and promezathine hydrochloride $25 \mathrm{mg}$. orally is desirable. Under local anæsthesia the brachial artery is exposed in the cubital fossa above the level of bifurcation into ulnar and radial arteries. A longitudinal incision is then made in the artery between two tape and polythene tourniquets. The right arm is used because the patient is placed in the left anterior oblique position and also because it is easier to manipulate the catheter tip in the root of the aorta from the right brachial artery. Adequate heparinization is essential to prevent thrombosis in the brachial artery and clot formation in the catheter tip. A dose of $2 \mathrm{mg}$. per $\mathrm{kg}$. body weight of heparin is given via the arterial catheter when the tip is at the level of the axilla. Clot formation in the catheter is also prevented by repeated flushing with heparinized saline.

The Sones' catheter (supplied by U.S.C.I.) is size 8 except for the terminal $7 \mathrm{~cm}$., which is size $5(1.6 \mathrm{~mm}$. external diameter); it has an end hole and four side holes within $1 \mathrm{~cm}$. of the tip. A rotary link and a threeway tap are connected to the other end of the catheter. Through one of the two inlets the catheter is linked by a polythene tube to a Cambridge manometer so that the pressure in the aorta or coronary orifice can be continuously observed on an oscilloscope. Through the second inlet to the catheter heparinized saline and contrast medium can be injected. The catheter is passed to the root of the aorta where the distal narrow portion readily loops. By rotating the patient to a $45^{\circ}$ ieft anterior oblique position superimposition of the aorta and spine is avoided and the orifices of the coronary arteries are separated, the left to the left-hand border, the right to the right-hand border of the aorta.

The catheter is screened through a 5 in. Philips image intensifier and double periscope viewer and manipulated towards one or other of the coronary ostia. If location of the right coronary ostium is difficult, further rotation of the patient to approximately $60^{\circ}$ in the same oblique may be helpful. Directly the position of the catheter tip in the coronary orifice is confirmed by a small (2-3 ml.) hand injection of opaque medium, the viewer is replaced by a $35 \mathrm{~mm}$. Arriflex camera. Further injections of medium are given in increasing doses up to about $8 \mathrm{ml}$. until the coronary artery is seen to be completely filled. Each injection is filmed at 50 frames a second and viewed through the camera shutter. This allows immediate visual control of the coronary angiogram. It is usually necessary to move the camera field as the medium flows down the coronary artery. In order to see the coronary arteries on the diaphragmatic surface of the heart it is essential to film the injection during inspiration. It is often necessary to give four to eight injections into each coronary artery with the patient in different degrees of obliquity in order to avoid overlap of the origins of the major branches. The true lateral position has been found useful in separating the proximal parts of the anterior descending and circumflex branches of the left coronary artery. Continuous monitoring of the electrocardiogram and coronary arterial pressure is maintained throughout the procedure. If the coronary arterial pressure drops, the catheter is immediately withdrawn from the coronary arterial as impaction for any length of time is liable to provide ventricular fibrillation.

The $35 \mathrm{~mm}$. film is processed commercially and returned within 24-48 hours together with a contact $35 \mathrm{~mm}$. copy and a $16 \mathrm{~mm}$. optically reduced copy. The contact $35 \mathrm{~mm}$. film is analysed in a Tage Arno editor viewer and the $16 \mathrm{~mm}$. film is projected in the usual way for demonstration to larger audiences.

\section{RESULTS}

Electrocardiographic Changes Resulting from Injection of Contrast Medium into Single Coronary Arteries. Marked T wave inversion or positive peaking is always recorded after injection unless the artery is small or severely diseased. Negative $\mathrm{T}$ wave changes are recorded in leads facing the myocardium through which medium circulates while positive changes are seen in leads facing the opposite side of the heart. This pattern has been consistently noted in leads aVL and aVF, and is not 


\section{|||||||||||||||||||||||||||||||||||||||||||||||||||| $\mid$}


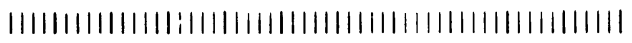

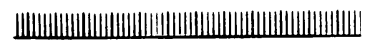

B

due to ischæmia but to the hypertonicity of the medium. The difference between ischæmia and perfusion with opaque medium was clearly demonstrated by Case 8 (Fig. 3A). This patient, aged 49, with angina pectoris for 4 years, had cardiographic evidence of inferior infarction ( $Q$ in III and aVF). On cannulation of the left coronary artery he experienced angina and the lead being observed at the time (aVL) showed S-T elevation (Fig. 3B). After prompt withdrawal of the catheter, angina and S-T elevation subsided. After a time the same artery was outlined by an injection of contrast medium injected in the sinus just below the orifice. There was no angina, the S-T segment (aVL) did not change, but steep T inversion occurred (Fig. 3C).

Failure to Outline the Coronary Arteries. In 2 patients it was impossible to pass the catheter from the innominate artery to the ascending aorta owing to a dilated and elongated aorta. In both cases the left arm was then used and the investigation successfully carried out.

Of our 25 cases, failure to cannulate one of the two coronary arteries occurred in 9: the left coronary artery in 6 , the right coronary in 3 . Inexperience with the technique was the most common cause of failure, 6 of the unsuccessful investigations occurring in the first 10 cases studied. Brachial artery spasm, resulting in poor mobility of the catheter within the aorta was a contributory factor in 2 of the other 3 cases. In one patient who came to autopsy (Case 9) an occlusion of the right coronary artery was found to begin within $5 \mathrm{~mm}$. of the orifice so that failure to cannulate it in life was understandable.

Because this investigation is of doubtful value unless each coronary artery is filled, every effort must be made to obtain an arteriogram from an injection of 10-15 ml. opaque medium given into the appropriate aortic sinus if cannulation of an artery is unsuccessful. Thus in 5 of our 9 cases a sinus injection has resulted in satisfactory coronary filling.

\section{COMPLICATIONS}

Ischamia of the Limb Consequent Upon Arteriotomy. In this department a follow-up study has been made of the peripheral pulse and limb function in the 120 patients submitted to arteriotomy for 
left heart catheterization since the beginning of 1961 . The pulse returned immediately or within 48 hours of catheterization in 106 patients $(88 \%)$. Of the remaining 14 patients mild ischæmic symptoms persisted in 4 for up to 3 months and in a fifth for 12 months. In this patient, a housewife aged 38 (Case 16 of the coronary arteriography series), ischæmic pain in the right forearm prevented her from performing sustained effort with this hand, such as peeling potatoes and vigorous brushing of the hair. In the other 4 patients, claudication was also the only manifestation of ischrmia encountered, but was so mild that it was only elicited by direct questioning.

Coronary Artery Obstruction. The catheter itself may obstruct a coronary artery. Johnson and Logan (1958) have stressed the danger of accidental coronary artery catheterization during aortic and left ventricular catheter and angiographic investigations. Serious obstruction is less likely to occur with the Sones' catheter because of its narrow tip, but in three patients angina has occurred with the tip in the left coronary orifice. In two others obstruction to a diminutive right coronary artery has only been recognized by the recording of a "wedged" coronary trace, i.e. a pressure pulse having an aortic form in systole and a ventricular form in diastole.

It is therefore essential for the electrocardiogram and aortic pulse tracing to be observed continuously throughout the procedure. Other possible causes of coronary occlusion include clot from the catheter tip and coronary artery dissection, which could conceivably result from the injection of contrast medium under too high a pressure into the arterial wall adjacent to an atherosclerotic plaque. These complications have not been encountered in this series.

Cardiac Arrest. Equipment necessary for external defibrillation and electrical stimulation should be available for immediate use at all times. Cardiac arrest has not been encountered during coronary arteriography but we have had occasion to use emergency resuscitation in this department in one case: a patient with aortic stenosis developed asystolic arrest following a left ventricular puncture but the immediate application of external pacemaker stimuli restored the circulation until natural recovery took place.

\section{Discussion}

Anatomical Considerations. A knowledge of the anatomy of the coronary arteries and their variations in origin and distribution is essential (Di Guglielmo and Guttadauro, 1954). While it is customary from an anatomical view point to refer to two (left and right) or three coronary arteries (anterior descending, circumflex, and right coronary artery), it is suggested that the three main groups of arteries supplying the left ventricle should be particularly studied in arteriography. These are: (1) the anterior descending supplying the anterior ventricular and interventricular septal myocardium, (2) the circumflex artery branches-in particular the artery to the obtuse margin and the median (or "third primary division")-all supplying the lateral free wall; and (3) the posterior interventricular artery supplying the diaphragmatic surface of the left ventricle and interventricular septum: the circumflex branch of either the left or the right coronary artery may supply this branch depending on which is the "dominant" artery. The most constant artery in size and origin is the anterior descending, and the large proportion of the left ventricle supplied by this artery has been stressed by James and Burch (1958). In 10-15 per cent of subjects there is a left dominant arterial pattern, the left coronary artery supplying parts of the right ventricle as well as all the left ventricle (Blumgart and Zoll, 1960). In patients in whom this pattern is recognized at the time of left coronary arteriography, demonstration of the right coronary artery is not essential. Furthermore, in two subjects in whom a diminutive right coronary artery was cannulated a "wedge" arterial trace was obtained, indicating that arteriography might have been dangerous.

A complete study of the left coronary artery is important, not only on anatomical grounds but also because atherosclerosis affects the first 2-4 cm. of the anterior descending artery more than any other portion of the coronary arterial tree. This has been recognized since Herrick wrote in 1912 that the "reputation of the descending branch of the left coronary as the artery of sudden death is not undeserved." We have come to regard an arteriogram to be of little value if the proximal part of this vulnerable artery is not clearly demonstrated. Unfortunately this may not be 


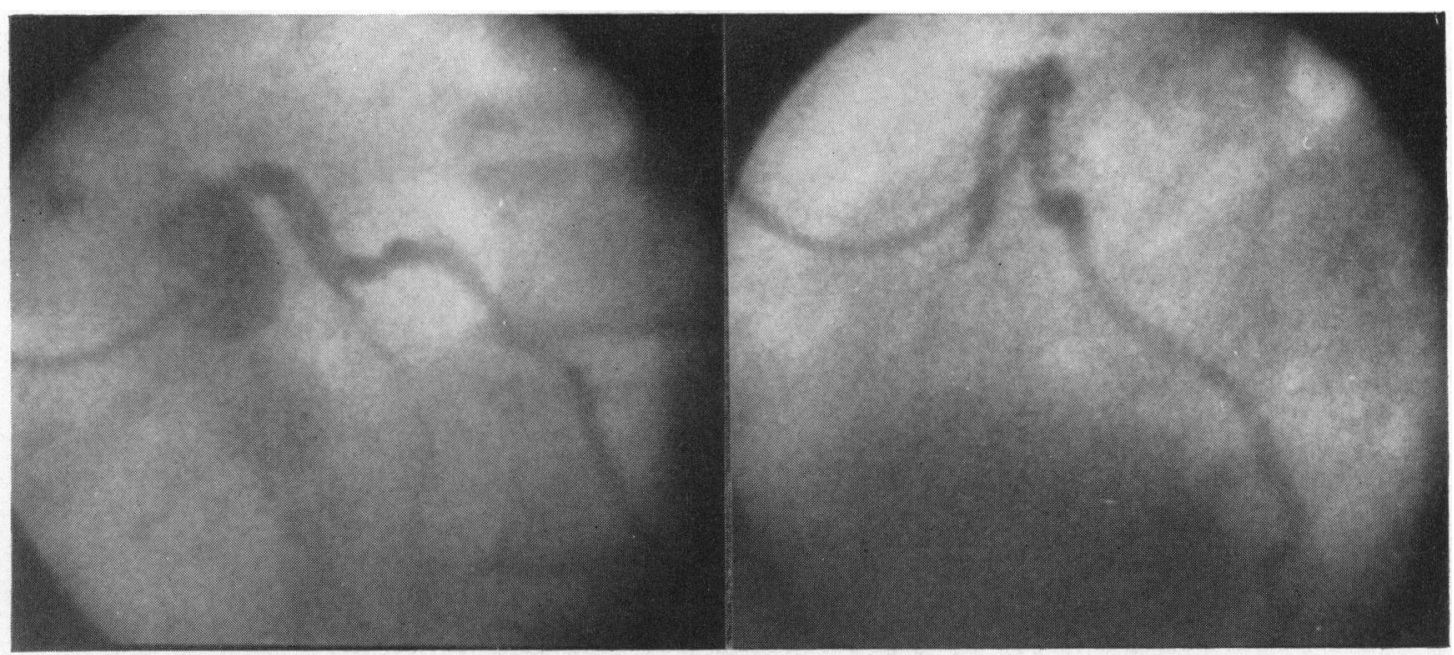

A

B

Fig. 4.-Case 10. Injection into the left coronary artery, (A) in the left anterior oblique, and (B) in the left lateral porjection. Superimposition of the anterior descending and circumflex arteries in the left anterior oblique projection has been overcome by rotation of the patient into the left lateral position. The anterior descending artery is almost completely occluded.

easy owing to overlapping with the circumflex artery and because the proximal part of the anterior descending artery is often seen "end-on" in the left anterior oblique projection. Overlapping of the beginnings of the anterior descending and circumflex arteries is well illustrated in Fig. 4 (Case 10). We therefore aim to film injections of the left coronary artery in the left anterior oblique and left lateral projections. The left anterior oblique position is ideal for the right coronary arteries. Some difficulty has been experienced in showing the posterior interventricular branches owing to superimposition of the diaphragm, but this may be overcome by filming in full inspiration.

2. Interpretation of Coronary Arteriograms. Some assessment of the accuracy of arteriography in demonstrating coronary disease is essential. When it is possible to demonstrate an occlusion (Case 10, Fig. 4 -anterior descending artery) or a severe narrowing (Case 6, Fig. 5-circumflex artery) such findings are clearly important. The reliability of arteriography in consistently demonstrating significant lesions must be considered. This problem was examined by Lehman (1959) who was impressed by the unreliability of coronary arteriography in dogs in detecting experimentally induced coronary artery lesions "resembling atherosclerosis." He stressed the importance of "delivering a sufficient amount and concentration of radio-opaque medium to effect good coronary artery filling." That a greater concentration of opaque medium in the coronary arteries is obtained with selective injection than with aortography is shown by Case 4 (Fig. 1 and 2). There are other advantages of selective coronary opacification when combined with cineradiography. The operator is able to film and view each injection of medium simultaneously and therefore to alter the degree of rotation of the patient or position of the catheter between injections. Thus superimposition of main branches may be overcome (Fig. 4, Case 10) and failure to opacify a main artery, which may result from a faulty position of the catheter, may be corrected. The latter point is illustrated by Case 15 (Fig. 6). Here the circumflex artery failed to fill with the catheter tip in the anterior descending artery (B and $\mathrm{C}$ ), but after withdrawal to a point proximal to the bifurcation (A), good filling of the circumflex artery was obtained.

Despite satisfactory coronary opacification it appears that in some circumstances coronary disease may be difficult to demonstrate and, if demonstrated, difficult to assess. This arises from the fact that a severely narrowed artery having a slit or crescentic shaped lumen will appear to be 


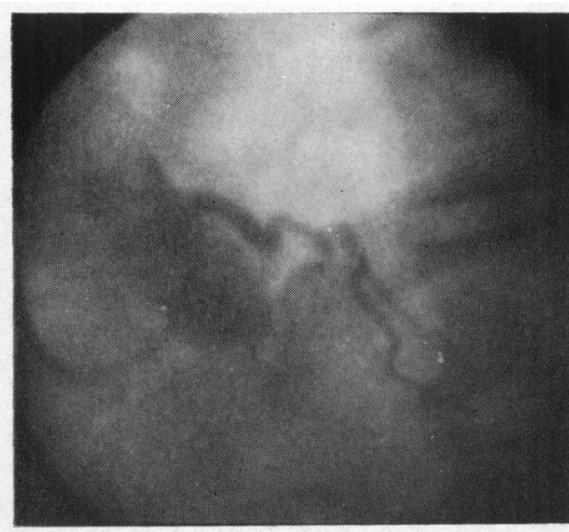

LEFT

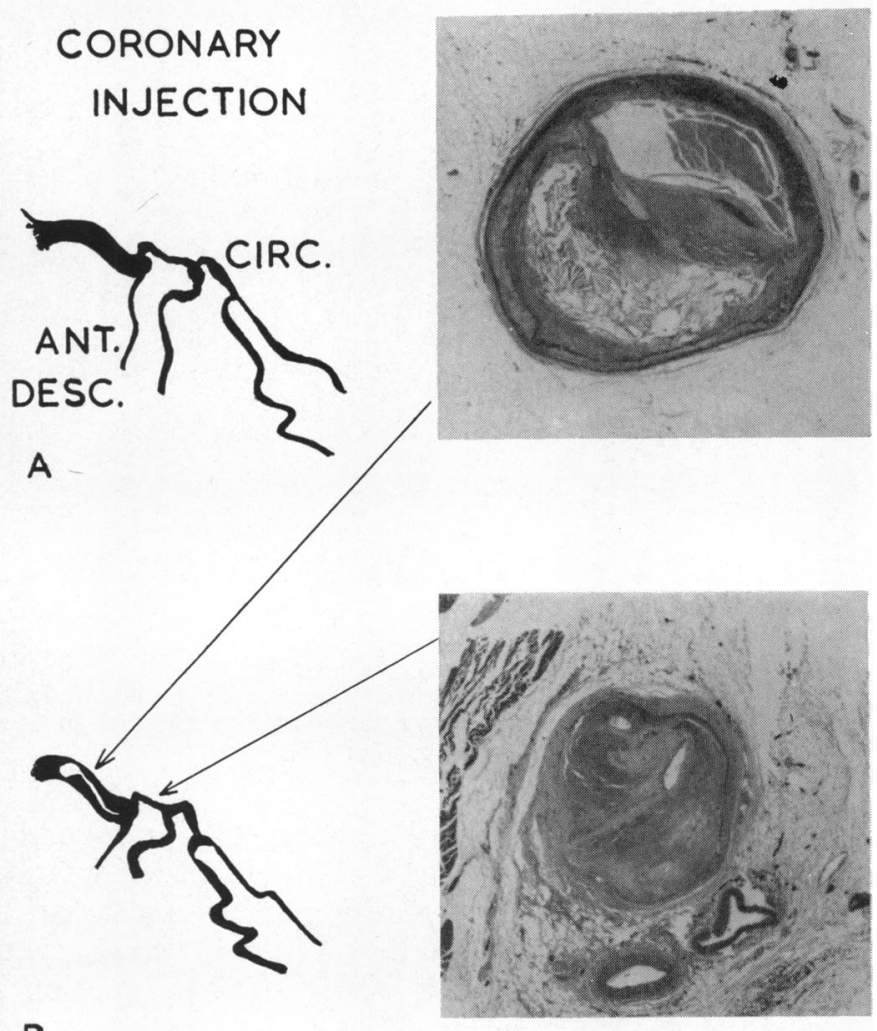

B

FIG. 5.-Case 6. (A) Left coronary arteriogram showing occlusion of the anterior descending artery and severe narrowing of the circumflex artery. (B) Left coronary arteriogram showing a filling defect and "split-lumen" sign in the trunk of the left coronary artery. This appearance was seen in only a few frames immediately after the end of each of several injections. Sections of the left coronary and circumflex arteries were obtained at necropsy at the sites indicated and show unexpectedly severe disease.

normal when viewed at right angles to the broad axis of the stenosis, providing there is good filling. Coronary arteriograms performed at autopsy have supported this point. Here the contrast is clearly much greater than is available in life. In one case a significant narrowing was almost overlooked because the artery appeared to have a normal lumen during most of the injection. In this patient, Case 15 (Fig. 6), a severe obstruction of the anterior descending artery had been expected on clinical grounds. He had had a typical severe anterior myocardial infarction two years previously followed by angina, and it was therefore likely that any flow observed at arteriography in the first part of the anterior descending artery would be through a recanalized thrombotic occlusion. This did not seem likely on initial inspection of the film (Fig. 6A and B) but careful study of the late phases of the film, frame by frame, revealed that there were indeed signs strongly suggesting a severe stenosis. These signs occurred immediately after the end of injection when the flow of medium was diminishing and were a spiral-shaped greatly narrowed lumen, and secondly, delay in clearance of medium immediately distal to this segment following all injections of the left coronary or anterior descending arteries. It is of interest that the spiral pattern of atherosclerotic disease in this artery has been noted by Enos, Holmes, and Beyer (1962) in autopsy studies.

3. Correlation with Necropsy Findings. Four patients have come to necropsy within six months 

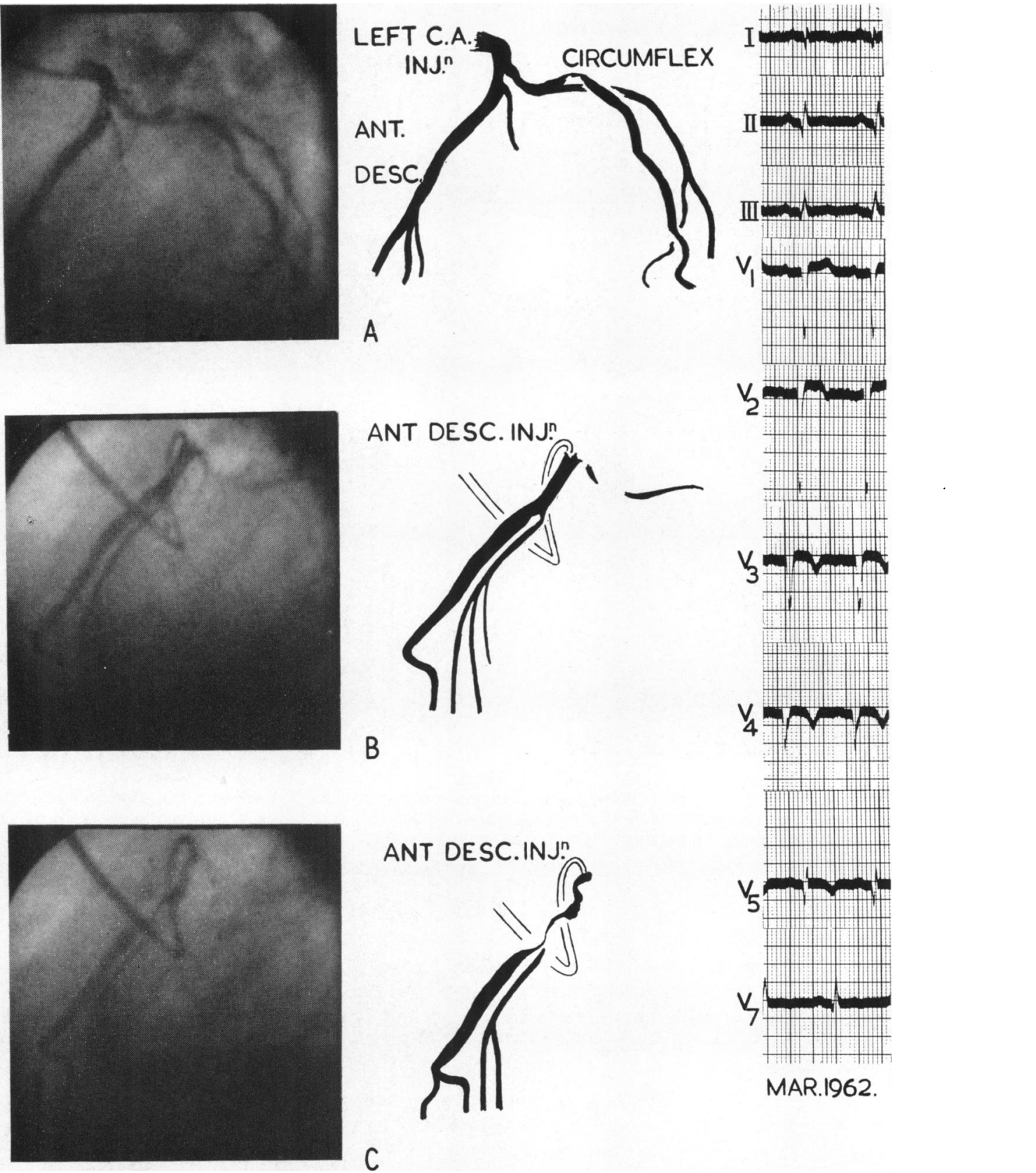

FIG. 6.-Case 15. (A) A frame from a ciné film taken during an injection into the left coronary artery. Left C.A. position, about $40^{\circ}$ left anterior oblique. B and $C$ frames from a ciné film (earlier than film depicted in (A) taken during and after, respectively, and injection into the anterior descending (ANT. DESC.) artery. Position, about $60^{\circ}$ left anterior oblique. The cardiogram at the time of coronary arteriography is shown on the right. This patient, a car dealer, aged 38 , presented 2 years before with anterior myocardial infarction. Subsequently he has been unable to work because of severe angina of effort. The cardiogram which showed $Q$ waves and steep $T$ inversion in the anterior $\mathrm{V}$ chest leads at the time of infarction has not changed. Note that a severe stenosis in the anterior descending artery was clearly outlined only in the late phase of the injection into this artery (see text). 

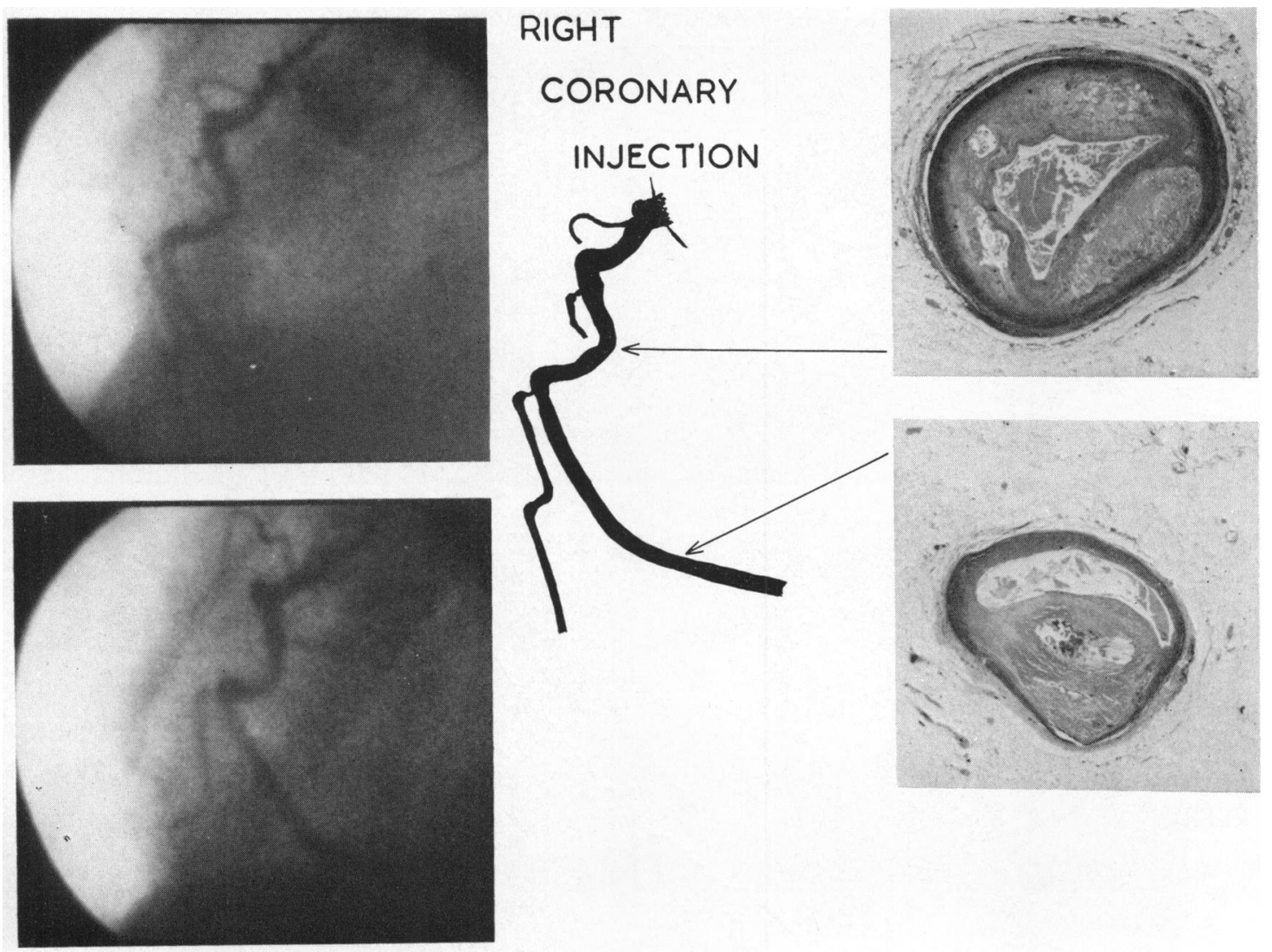

Fig. 7.-Case 6. Right coronary arteriogram showing minor irregularities of the lumen near the origin of a marginal artery which is severely narrowed at its beginning. At necropsy severe confluent atheroma was found along the entire length of the right coronary artery. This degree of atherosclerosis was not predicted by coronary arteriography.

of coronary arteriography and in all the estimated age of the disease found was such that valid comparative observations were possible. The chief conclusion from this study (to be reported in detail elsewhere) was that arteriography in life may underestimate the extent of disease. Consequently if in doubt the worst possible assessment of a lesion demonstrated in life is probably the most accurate. This is well shown in Case 6 (Fig. 5 and 7). This patient, a man of 54 years, had a two-year history of progressively increasing angina with effort and, more recently, at rest. Coronary arteriography showed occlusion of the anterior descending artery and severe narrowing of the circumflex artery (Fig. 5). Because the main left coronary trunk appeared normal (Fig. 5) and the right coronary only slightly diseased (Fig. 7), he was recommended for endarterectomy of the anterior descending artery, a procedure that proved fatal. The severity of the coronary disease found at necropsy five months after coronary arteriography was in excess of that expected (Fig. 5B). The left coronary artery proximal to the bifurcation had been thought to be normal and only on retrospective frame-by-frame examination of the late phases of four successive arteriograms was it appreciated that signs of disease were present. Note the proximal filling defect continuing distally as a "split lumen" (Fig. 5B). On review of all arteriograms this characteristic appearance was noted either transiently or throughout each film in five other patients (Cases 5, 10,11,14, and 15), in all of whom severe disease was suspected on other grounds. It is presumed that the split lumen appearance is due to blood clearing opaque medium from the central parts of the lumen with medium persisting 
in the more slowly flowing recesses bordering atheromatous plaques. Nevertheless in some cases this appearance may be mistaken for laminar flow in a normal artery (Dotter and Frische, 1958). The value of the split lumen sign is therefore much greater if associated with other signs of disease in the same artery.

The disease in the right coronary artery of this patient demonstrated in Fig. 7 was also found at necropsy to be in excess of what was predicted. The sections illustrated are representative of the degree of narrowing produced by confluent but eccentric atherosclerotic plaques observed from the level of origin of the marginal artery to beyond the crux into the posterior interventricular artery. While the presence of some disease was deduced from the irregular outline of the aortogram near the origin of the marginal artery, the absence of further irregularities and an apparent intact lumen distally led us to believe that important disease was absent.

\section{SUMMARY}

Selective coronary arteriography with cineradiography is, we suggest, the most reliable technique available for coronary arteriography.

We have not experienced serious complications in the 25 patients investigated. Although some difficulty with the technique was experienced initially, with practice satisfactory arteriograms have been obtained consistently. It is normally not time-consuming and may therefore be combined with right and left heart catheterization and angiocardiography.

From anatomical and pathological considerations, the importance of demonstrating clearly the left coronary artery and in particular the anterior descending artery is stressed.

Factors relating to interpretation of films are discussed. From necropsy experience it has been shown that severe coronary disease pathologically may be difficult to detect by coronary arteriography in life. Some reasons for this and means to improve interpretation are suggested.

We wish to thank Dr. Aubrey Leatham for his help with this paper and the Technical and Radiological Staffs for their invaluable assistance during the coronary angiograms. We are grateful to Miss Victoria McClure for typing the manuscript.

\section{REFERENCES}

Arnulf, G., and Buffard, P. (1959). L'artériographie des coronaires grace à l'acétylcholine. Documents expérimentaux et cliniques. Ann. Radiol., 2, 685.

Bilgutay, A. M., and Lillehei, C. W. (1962). New method for coronary arteriography. J. Amer. med. Ass., 180, 1095.

Blumgart, H. L., and Zoll, P. M. (1960). Pathologic physiology of angina pectoris and acute myocardial infarction. Circulation, 22, 301.

Di Guglielmo, L., and Guttadauro, M. (1954). Anatomie variations in the coronary arteries. Acta radiol. (Stockh.), 41, 393.

Dotter, C. T., and Frische, L. H. (1958). Visualization of the coronary circulation by occlusion aortography: a practical method. Radiology, 71, 502.

Enos, W. F., Holmes, R. H., and Beyer, J. C. (1962). Pathology of coronary arteriosclerosis. Amer. J. Cardiol., 9,343 .

Gensini, G. G., Di Giorgi, S., and Black, A. (1961). New approaches to coronary arteriography. Angiography, 12, 223.

Herrick, J. B. (1912). Clinical features of sudden obstruction of the coronary arteries. J. Amer. med. Ass., 59, 2015. James, T. N., and Burch, G. E. (1958). Blood supply of the human interventricular septum. Circulation, 17, 391.

Johnson, A. M., and Logan, W. D. (1958). Coronary artery catheterization during thoracic aortography. Brit. Heart J., 20, 411.

Lang, E. K., and Sabiston, D. C. (1961). Coronary arteriography in the selection of patients for surgery. Radiology, 76, 32 .

Lehman, J. S. (1959). Coronary arteriography: Practical considerations. Progr. cardiovasc. Dis., 2, 36.

, Boyer, R. A., and Winter, F. S. (1959). Coronary arteriography. Amer. J. Roentgenol., 81, 749.

Michell, G., and Jefferson, K. (1962). Angiography of the coronary circulation in living dogs using timed diastolic injections. Brit. Heart J., 24, 11.

Nordenström, B., Ovenfors, C., and Törnell, G. (1962). Coronary angiography in 100 cases of ischemic heart disease. Radiology, 78, 714.

Radner, S. (1945). Attempt at roentgenologic visualization of coronary blood vessels in man. Acta radiol. (Stockh.), 26, 497. 
Sloman, G., and Jefferson, K. (1960). Cine-angiography of the coronary circulation in living dogs. Brit. Heart J., $22,54$.

Sones, F. M. (1960). Cinecardioangiography. In Clinical Cardiopulmonary Physiology, 2nd ed., ed. B. L. Gordon, p. 136. Grune and Stratton, New York.

, and Shirey, E. K. (1962). Cine coronary arteriography. Mod. Conc. cardiovasc. Dis., 31, 735.

Tapia, F. A., Bolton, H. E., and Mazel, M. S. (1961). Selective coronary cine-arteriography: an improved method for visualization of the coronary arteries. Angiology, 12, 46.

Thal, A. P., Richards, L. S., Greenspan, R., and Murray, M. J. (1958). Arteriographic studies of the coronary arteries in ischemic heart disease. J. Amer. med. Ass., 168, 2104.

Williams, J. A., Littmann, D., Hall, J. H., Bellman, S., Lambert, P. B., and Frank, H. A. (1960). Coronary arteriography. II. Clinical experiences with the loop-end catheter. New Engl.J. Med., 262, 328. 